\title{
Antiplatelet agents used for early intervention in acute coronary syndrome: Myocardial salvage versus bleeding complications
}

\author{
Sary F. Aranki, MD, ${ }^{\mathrm{a}}$ and Simon C. Body, $\mathrm{MBChB}, \mathrm{MPH}^{\mathrm{b}}$
}

\section{Supplemental material is available online.}

Acute coronary syndrome (ACS) is a costly health care issue worldwide. ${ }^{1}$ Numerous well-conducted clinical trials have demonstrated that early intervention is associated with the greatest long-term survival, better myocardial preservation, and improved quality of life. Consequently, inpatient cardiovascular procedures in the United States have increased over 4-fold since $1979 .{ }^{1}$ Notably, the use of antiplatelet therapies has increased well beyond this number and has affected the management of the one-quarter million patients who undergo cardiac surgery each year. The use of antithrombotic agents carries a risk of minor and major bleeding, notably when patients undergo coronary artery bypass grafting (CABG) in the early phase of ACS, the time at which antithrombotic use is greatest and most effective. Concerns over excess bleeding in patients undergoing CABG may lead to withholding antiplatelet agents, perhaps sacrificing early benefit for some patients. The use of blood products, notably platelet transfusions, to treat bleeding has short- and longterm risks that have not yet been balanced against the risks of decreased myocardial survival associated with nonuse of antiplatelet therapy. A better understanding of the risks and benefits associated with early antiplatelet therapy is necessary to improve the outcomes of all patients with ACS, including those who undergo $\mathrm{CABG}$.

\section{VALUE OF EARLY INTERVENTION IN ACS}

Numerous clinical trials conducted over 3 decades have rigorously evaluated management strategies for ACS. These studies have shown that early intervention combined with appropriate antiplatelet agent use results in significantly improved outcomes. The findings of these trials have been

\footnotetext{
From the Division of Cardiac Surgery ${ }^{\mathrm{a}}$ and the Department of Anesthesiology, Perioperative and Pain Medicine, ${ }^{\mathrm{b}}$ Brigham and Women's Hospital, Boston, Mass. This commentary was written and edited by the authors, who take full responsibility for its content. Editorial assistance with searching the literature, coordinating revisions, and creating the figure and tables in preparation of this manuscript was provided by Melanie Leiby, $\mathrm{PhD}$, and funded by the Bristol-Myers Squibb/Sanofi Pharmaceutical Partnership. Drs Aranki and Brody did not receive any compensation for this work.

Received for publication Jan 6, 2009; revisions received March 26, 2009; accepted for publication April 11, 2009.

Address for reprints: Sary Aranki, MD, Division of Cardiac Surgery, Brigham and Women's Hospital, 75 Francis St, Boston, MA 02115 (E-mail: saranki@partners. org).

J Thorac Cardiovasc Surg 2009;138:807-10

$0022-5223 / \$ 36.00$

Copyright (c) 2009 by The American Association for Thoracic Surgery

doi:10.1016/j.jtcvs.2009.04.052
}

used to generate the well-stated and widely used American College of Cardiology/American Heart Association (ACC/ AHA) clinical guidelines for ACS management. ${ }^{2,3}$

Results of trials such as FRISC-II (Fast Revascularization during Instability in Coronary artery disease-II), ${ }^{4}$ ISARCOOL (Intracoronary Stenting with Antithrombotic Regimen COOLing off), ${ }^{5}$ and ACUITY (Acute Catheterization and Urgent Intervention Triage strategY) ${ }^{6}$ exemplify the value of antiplatelet drugs in early ACS treatment. However, the value of early coronary intervention accompanied by profound inhibition of platelet aggregation and thrombus formation incurs an increased risk of bleeding, which has its own attendant morbidity and mortality. Despite the additional risk of bleeding, the use of appropriate pharmacotherapy in patients with ACS is paramount. Recognition of both the benefit of early interventional therapy in patients with ACS with the simultaneous finding of benefit for antiplatelet therapies has led to critical issues in developing strategies that best combine these tactics while minimizing potential risk. Central to this discussion is the following paradox: cardiologists want intensive antiplatelet therapy, frequently with multiple agents, to avoid thrombosis whereas surgeons want to minimize antiplatelet therapy to avoid bleeding. Thus, there is tension in balancing the risks of bleeding and transfusion against improved long-term outcomes in a frequently used treatment pattern for patients with ACS consisting of early intervention and later CABG. Although significant evidence exists to address this concern, it is unclear whether the perception of bleeding risk with antiplatelet agents is aligned with actual evidence for the real risk-versus-benefit equation.

\section{PLATELET INHIBITION AND THE RISK OF BLEEDING}

A wealth of clinical trial data provides evidence for the benefit of antiplatelet therapy in preventing recurrent ischemia after ACS. ${ }^{7,8}$ This is a critical consideration in secondary prevention, particularly in the perioperative period when platelet activity is high and associated with significant risk for a second, potentially fatal ischemic event. It is therefore imperative that a timely antiplatelet strategy be implemented with due consideration for the potential and real risk of abruptly stopping such beneficial therapy because of bleeding concerns in patients undergoing CABG.

Intrinsic to any strategy for inhibiting platelet function is the increased risk for bleeding. Bleeding is usually classified according to clinical or laboratory criteria as major (life-threatening, severe) or minor. ${ }^{9}$ However, bleeding 


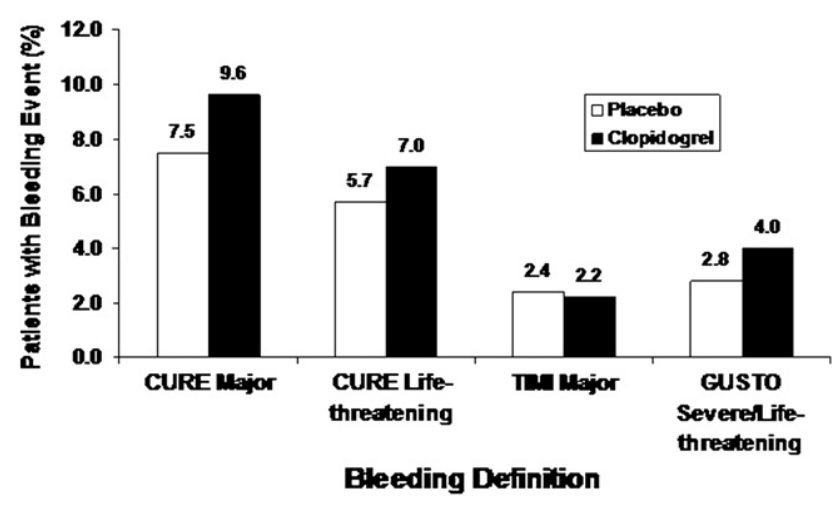

FIGURE 1. Differences in risk of bleeding in patients who underwent coronary artery bypass grafting in the Clopidogrel in Unstable angina to prevent Recurrent Events (CURE) trial depending on the bleeding definition used. All $P$ values $>$.05. GUSTO, Global Utilization of Streptokinase and Tissue Plasminogen Activator for Occluded Coronary Arteries; TIMI, Thrombolysis In Myocardial Infarction. Adapted with permission. ${ }^{10}$

definitions have not been uniformly applied, leading to as much as a 3 -fold difference in the reported incidence of major bleeding (Figure 1). ${ }^{10}$ Points of discrepancy between criteria for major bleeding include a decrease in hemoglobin (from $\geq 3 \mathrm{~g} / \mathrm{dL}$ to $\geq 5 \mathrm{~g} / \mathrm{dL}$ ), a requirement for transfusion $(\geq 1$ unit or $\geq 2$ units), or a requirement for an overt source of bleeding. ${ }^{10}$

A growing body of evidence shows that, regardless of the classification system applied, bleeding is an independent predictor of adverse clinical outcomes. TIMI (Thrombolysis In Myocardial Infarction) major bleeding, GUSTO (Global Utilization of Strategies To Open occluded arteries) moderate or severe bleeding, and a drop in hemoglobin have all been associated with early mortality and/or other adverse cardiovascular events within 30 days ${ }^{10}$; whether such bleeding is also associated with mortality at 6 months or 1 year is currently unclear. Thus, the prevalence and risk for bleeding in patients with ACS with or without intervention, remains a significant concern requiring careful consideration to balance the risk of bleeding with the benefit of antiplatelet therapies.

\section{ANTIPLATELET THERAPIES AND INTERVENTION}

Based on the importance of antiplatelet therapy in treating ACS, evidence-based guidelines for their use have been prepared. ${ }^{2,3}$ Because aspirin and clopidogrel are the only antiplatelet therapies currently recommended at a class I level for both immediate and long-term use in patients with ACS they will be the main focus of the remaining discussion.

Historically, the benefits of early aspirin use in the treatment of patients with ACS have been well established. ${ }^{11}$ For patients undergoing $\mathrm{CABG}$ preoperative aspirin use has been associated with improved graft patency and reduces the risk of perioperative myocardial infarction (MI), in-hospital mortality, and other adverse postoperative outcomes (Table E1).

Older aspirin studies sparked debate around aspirin use in $\mathrm{CABG}$ inasmuch as they showed that preoperative aspirin use was associated with increased blood loss, greater transfusion rates, and higher re-exploration rates (Table E2). However, numerous subsequent studies have described no significant increase in bleeding complications in patients undergoing $\mathrm{CABG}$ receiving aspirin in the 7 days before surgery (Tables E2 and E3). Thus, aspirin is not a significant risk factor for bleeding or transfusion.

The efficacy of dual antiplatelet therapy with aspirin and clopidogrel has been compared with aspirin monotherapy in patients with ACS and those undergoing percutaneous coronary intervention (PCI). The results of these large, prospective, randomized trials show that adding clopidogrel to aspirin consistently reduces the risk of adverse events, in most cases, without significantly increasing the risk of bleeding (Table E4). Overall, there is a clear, consistent benefit associated with clopidogrel pretreatment before PCI, and the reduction in cardiovascular events before PCI suggests that clopidogrel pretreatment should be initiated as soon as possible. Although the risk of bleeding may be greater with dual antiplatelet therapy, the excess risk is not enough to outweigh the benefits.

Data also suggest that aspirin and clopidogrel improve clinical outcomes without being associated with increased bleeding after CABG. ${ }^{12,13}$ Among the 1013 patients in the CURE (Clopidogrel in Unstable angina to prevent Recurrent Events) study who received CABG during their index hospitalization, dual antiplatelet therapy reduced the relative risk of cardiovascular death, MI, and stroke by $19 \%$ compared with patients who received aspirin alone (relative risk, $0.81 ; 95 \%$ confidence interval $[\mathrm{CI}], 0.59-1.12)$; the bulk of this benefit was due to an $18 \%$ reduction in the relative risk of events occurring before surgery (relative risk, 0.82 ; 95\% CI, 0.58-1.16). ${ }^{12}$ Among the 136 CLARITY (Clopidogrel as Adjunctive ReperfusIon TherapY) patients who underwent $\mathrm{CABG}$, dual therapy with aspirin and clopidogrel reduced the risk of cardiovascular death, MI, and recurrent ischemia by $34 \%$ compared with those who received aspirin (odds ratio, 0.66; 95\% CI, 0.27-1.63), a benefit due entirely to a reduction in $\mathrm{MI}$ and recurrent ischemia before surgery (odds ratio, 0.46; 95\% CI, 0.16-1.36). ${ }^{13}$ Results of other studies disagree with those of CURE and CLARITY inasmuch as they show an increased risk of procedural bleeding, re-exploration, blood product usage, and chest drain blood loss in patients undergoing CABG receiving clopidogrel and aspirin, particularly within 5 to 7 days of the operation (Tables E2 and E3).

\section{THE CABG CONUNDRUM}

Only $7 \%$ to $12 \%$ of patients with non-ST-elevation MI and $4 \%$ of patients with ST-elevation MI undergo 
CABG. ${ }^{14,15}$ Further, fewer than $1 \%$ of all CABG operations are done on an emergency basis (immediately after failed $\mathrm{PCI}){ }^{16}$ Given that CABG is performed in only a minority of patients, is effective antiplatelet treatment being withheld from the majority for the benefit of the minority?

The preponderance of evidence indicates that clopidogrel exposure within 5 to 7 days of CABG is associated with excess bleeding (Tables E2 and E3); thus, current guidelines recommend a clopidogrel washout period of 5 to 7 days before planned CABG. ${ }^{2,3}$ However, it is not clear how clopidogrel-associated excess bleeding has affected CABG clinical practice. Although transfusion rates are driven by institutional and practitioner preferences, they do provide some estimation of bleeding after CABG surgery. Thus, one way to loosely assess the effect of escalated platelet inhibition on $\mathrm{CABG}$ clinical practice is to examine the transfusion requirements reported for different antiplatelet regimens (Tables E2 and E3). Caveats to such comparisons include the potential masking effect of advancing surgical techniques ${ }^{17}$ the varying transfusion thresholds among hospitals, ${ }^{18}$ the nonhomogeneous use of antiplatelet regimens perioperatively, ${ }^{19}$ and physician biases toward prophylactic, and perhaps unnecessary, transfusion of plasma and platelets in clopidogrel-treated patients. ${ }^{18}$ Given these caveats, observational data suggest that transfusion rates for patients undergoing CABG have remained relatively unchanged in the dual antiplatelet therapy era. In the early days of CABG, almost all patients received a transfusion. ${ }^{20}$ With the advent of hemodilution and concerns over the quality of the blood supply, efforts were made to reduce blood transfusions throughout the 1980s and beyond. Examination of the data shows that although the amount of blood loss has increased anywhere from 30\% to $100 \%$ along with escalated platelet inhibition, the percentage of patients receiving transfusions (between 20\% and $60 \%$ depending on the study) and the amount of blood transfused per patient (mean of 2 to 3 units) has remained approximately the same (Tables E2 and E3). For this issue to be addressed more effectively, however, there is a need for prospective collection of post-CABG bleeding and transfusion rates in all clinical trials of antiplatelet agents.

The percentage of patients who have an adverse myocardial event while waiting for clopidogrel washout is not known. Although data from some studies support the CURE and CLARITY findings that fewer clopidogrelexposed patients have adverse outcomes, data from other studies suggest the opposite. ${ }^{19,21}$ It is possible to extrapolate data from PCI-CURE, in which the time to PCI was 6 days and the incidence of an MI while waiting for intervention was $5.1 \%$ in the placebo group and $3.6 \%$ in the clopidogrel group $(P=.04$; number needed to treat $=66$ to prevent an MI before PCI). Such an extrapolation suggests that the reduction in blood loss, risk of re-exploration, and blood product usage that accompanies cessation of clopidogrel for 5 to 7 days before CABG comes at the expense of approximately a $1 \%$ increase in the risk of MI while waiting for surgery. ${ }^{22}$ This rate may be highest in the period immediately after the first ACS event and may decline in the weeks or months that precede elective CABG.

Whether a reduced risk of bleeding is worth an increased risk of additional ischemic events remains an issue for continued debate. Adding to this debate are important considerations regarding the direct and indirect economic and social costs of hospitalization on patients and their families resulting from potentially unnecessary delays in CABG. In one study comparing resource use in a CABG patient cohort, the costs for those who underwent nonelective $\mathrm{CABG}$ were $33 \%$ higher than the costs for those who underwent elective CABG, a difference due almost entirely to costs incurred during a longer preoperative length of stay. ${ }^{23}$ Prolonged lengths of stay also expose patients, particularly those who are elderly, to the development of comorbid conditions related to long hospital stays, including decubitus ulcers (bed sores) and nosocomial infections. One way to limit the overall impact of a clopidogrel washout period may be to discharge patients to home, as results of a retrospective analysis of 125 patients who received CABG within 3 to 5 days of clopidogrel cessation showed that those discharged to home had similar intraoperative and postoperative outcomes as those who remained hospitalized. ${ }^{24}$ Overall, additional information on the risks of clopidogrel washout before CABG is needed.

\section{FUTURE DIRECTIONS}

The absence of reliable, easy, and quick platelet function testing for patients receiving a wide variety of antiplatelet drugs is a serious problem. There are several commercially available platelet function tests, but the overall utility of platelet function testing is limited by poor reproducibility and comparability among tests. Variability in response to antiplatelet agents, perhaps especially marked for the prodrugs, makes estimation of dosing and timing of antiplatelet administration very difficult. Furthermore, if physicians were able to more precisely determine which patients were at the greatest risk of bleeding, as well as the time at which antiplatelet-exposed patients could be safely operated on, appropriate upstream initiation of therapy might increase. For such a scenario to come to fruition, a single reproducible and inexpensive test to measure platelet inhibition is necessary.

\section{CONCLUSION}

Antiplatelet therapy is a mainstay of care for patients with ACS, and the majority of these patients benefit from its early use, as evidenced by a reduced risk of cardiovascular events while awaiting interventional revascularization. The vast majority of patients with ACS receive PCI as opposed to 
CABG; therefore, waiting to confirm coronary anatomy before initiating antiplatelet therapy would be to the detriment of $90 \%$ to $95 \%$ of patients if one considers that only $5 \%$ to $12 \%$ of patients with ACS undergo CABG. ${ }^{14,15}$

Although antiplatelet therapy is associated with clinical benefit in patients undergoing $\mathrm{CABG}$, it also increases the risk of bleeding in some, but not all, patients. Although prolonged cessation of clopidogrel in patients undergoing CABG before the procedure is associated with a reduction in perioperative blood loss and blood product usage and a reduced risk of re-exploration, these benefits come at the expense of a $1 \%$ increase in the risk of MI while awaiting surgery. ${ }^{22}$ Inasmuch as previous bleeding concerns associated with aspirin use before $\mathrm{CABG}$ have been overcome with increased clinical experience, the risk of bleeding with dual antiplatelet therapy may be reduced with increased operator experience and adjunctive therapeutic agents such as the lysine analogs to reduce surgical bleeding. Overall, strategies aimed at reducing the risk of bleeding should not compromise the clinical benefit of antiplatelet therapy. In the future, platelet function testing may increase the use of appropriate upstream antiplatelet therapy by helping surgeons determine when the risk of bleeding is lessened in antiplatelet-exposed patients.

\section{References}

1. Rosamond W, Flegal K, Furie K, Go A, Greenlund K, Haase N, et al. Heart disease and stroke statistics-2008 update: a report from the American Heart Association Statistics Committee and Stroke Statistics Subcommittee. Circulation. 2008;117: e25-146.

2. Anderson JL, Adams CD, Antman EM, Bridges CR, Califf RM, Casey DE Jr, et al. ACC/AHA 2007 guidelines for the management of patients with unstable angina/non ST-elevation myocardial infarction: a report of the American College of Cardiology/American Heart Association Task Force on Practice Guidelines (Writing Committee to Revise the 2002 Guidelines for the Management of Patients With Unstable Angina/Non ST-Elevation Myocardial Infarction): developed in collaboration with the American College of Emergency Physicians, the Society for Cardiovascular Angiography and Interventions, and the Society of Thoracic Surgeons: endorsed by the American Association of Cardiovascular and Pulmonary Rehabilitation and the Society for Academic Emergency Medicine. Circulation. 2007;116:e148-304.

3. Antman EM, Hand M, Armstrong PW, Bates ER, Green LA, Halasyamani LK, et al. 2007 focused update of the ACC/AHA 2004 guidelines for the management of patients with ST-elevation myocardial infarction: a report of the American College of Cardiology/American Heart Association Task Force on Practice Guidelines. J Am Coll Cardiol. 2008;51:210-47.

4. Lagerqvist B, Husted S, Kontny F, Stahle E, Swahn E, Wallentin L. 5-year outcomes in the FRISC-II randomised trial of an invasive versus a non-invasive strategy in non-ST-elevation acute coronary syndrome: a follow-up study. Lancet. 2006;368:998-1004.

5. Neumann FJ, Kastrati A, Pogatsa-Murray G, Mehilli J, Bollwein H, Bestehorn HP, et al. Evaluation of prolonged antithrombotic pretreatment ("cooling-off" strategy) before intervention in patients with unstable coronary syndromes: a randomized controlled trial. JAMA. 2003;290:1593-9.
6. Stone GW, McLaurin BT, Cox DA, Bertrand ME, Lincoff AM, Moses JW, et al. Bivalirudin for patients with acute coronary syndromes. N Engl J Med. 2006;355: 2203-16.

7. Antithrombotic Trialists' Collaboration. Collaborative meta-analysis of randomised trials of antiplatelet therapy for prevention of death, myocardial infarction, and stroke in high risk patients. BMJ. 2002;324:71-86.

8. Bowry AD, Brookhart MA, Choudhry NK. Meta-analysis of the efficacy and safety of clopidogrel plus aspirin as compared to antiplatelet monotherapy for the prevention of vascular events. Am J Cardiol. 2008;101:960-6.

9. Eikelboom JW, Hirsh J. Bleeding and management of bleeding. Eur Heart J Suppl. 2006;8:G38-45.

10. Steinhubl SR, Kastrati A, Berger PB. Variation in the definitions of bleeding in clinical trials of patients with acute coronary syndromes and undergoing percutaneous coronary interventions and its impact on the apparent safety of antithrombotic drugs. Am Heart J. 2007;154:3-11.

11. Cannon CP, Mehta SR, Aranki SF. Balancing the benefit and risk of oral antiplatelet agents in coronary artery bypass surgery. Ann Thorac Surg. 2005;80:768-79.

12. Fox KA, Mehta SR, Peters R, Zhao F, Lakkis N, Gersh BJ, et al. Benefits and risks of the combination of clopidogrel and aspirin in patients undergoing surgical revascularization for non-ST-elevation acute coronary syndrome: the Clopidogrel in Unstable angina to prevent Recurrent ischemic Events (CURE) Trial. Circulation. 2004;110:1202-8

13. McLean DS, Sabatine MS, Guo W, McCabe CH, Cannon CP. Benefits and risks of clopidogrel pretreatment before coronary artery bypass grafting in patients with ST-elevation myocardial infarction treated with fibrinolytics in CLARITY-TIMI 28. J Thromb Thrombol. 2007;24:85-91.

14. CRUSADE Quarter 4, 2006 Results. 2007 [cited 2007 December 3, 2007]; Available from: http://crusadeqi.com/Main/Ecab/Slides/CRUSADEResults2006Q4.ppt\#343,16, Slide16.

15. Fox KA, Anderson FA Jr, Dabbous OH, Steg PG, Lopez-Sendon J, Van de Werf F, et al. Intervention in acute coronary syndromes: do patients undergo intervention on the basis of their risk characteristics? The Global Registry of Acute Coronary Events (GRACE). Heart. 2007;93:177-82.

16. Yang EH, Gumina RJ, Lennon RJ, Holmes DR Jr, Rihal CS, Singh M. Emergency coronary artery bypass surgery for percutaneous coronary interventions: changes in the incidence, clinical characteristics, and indications from 1979 to 2003. J Am Coll Cardiol. 2005;46:2004-9.

17. Mack MJ. Beating heart surgery: does it make a difference? Am Heart Hosp J. 2003;1:149-57.

18. Snyder-Ramos SA, Mohnle P, Weng YS, Bottiger BW, Kulier A, Levin J, et al. The ongoing variability in blood transfusion practices in cardiac surgery. Transfusion. 2008;48:1284-99.

19. Mehta RH, Roe MT, Mulgund J, Ohman EM, Cannon CP, Gibler WB, et al. Acute clopidogrel use and outcomes in patients with non-ST-segment elevation acute coronary syndromes undergoing coronary artery bypass surgery. J Am Coll Cardiol. 2006;48:281-6.

20. Bracey AW, Radovancevic R. The hematologic effects of cardiopulmonary bypass and the use of hemotherapy in coronary artery bypass grafting. Arch Pathol Lab Med. 1994;118:411-6.

21. Pickard AS, Becker RC, Schumock GT, Frye CB. Clopidogrel-associated bleeding and related complications in patients undergoing coronary artery bypass grafting. Pharmacotherapy. 2008;28:376-92.

22. Mehta SR, Yusuf S, Peters RJ, Bertrand ME, Lewis BS, Natarajan MK, et al. Effects of pretreatment with clopidogrel and aspirin followed by long-term therapy in patients undergoing percutaneous coronary intervention: the PCI-CURE study. Lancet. 2001;358:527-33.

23. Violette PD, Filion KB, Haider S, Pilote L, Eisenberg MJ. A cost analysis of nonelective coronary artery bypass graft surgery. J Card Surg. 2006;21:621-7.

24. Kuchulakanti P, Kapetanakis EI, Lew R, Rha SW, Cheneau E, Satler LF, et al. Impact of continued hospitalization in patients pre-treated with clopidogrel prior to coronary angiography and undergoing coronary artery bypass grafting. J Invasive Cardiol. 2005; 17:5-7. 
TABLE E1. Benefit of aspirin after CABG

\begin{tabular}{|c|c|c|c|c|}
\hline Study & Patients & $\begin{array}{c}\text { Outcome in } \\
\text { ASA recipients }\end{array}$ & $\begin{array}{c}\text { Outcome in ASA } \\
\text { nonrecipients }\end{array}$ & Risk reduction \\
\hline Goldman et al ${ }^{\mathrm{E} 1}$ & $\begin{array}{l}406 \text { men who } \\
\text { underwent elective } \\
\text { CABG followed by } \\
\text { coronary angiography } \\
\text { at } 1 \text { year (299 received } \\
\text { ASA, } 107 \text { did not) }\end{array}$ & $\begin{array}{l}15.8 \% \text { of grafts were } \\
\text { occluded on angiography }\end{array}$ & $\begin{array}{l}22.6 \% \text { of grafts were } \\
\text { occluded on angiography } \\
(P=.029 \text { vs those } \\
\text { who received ASA })\end{array}$ & Not reported \\
\hline Dacey et $\mathrm{al}^{\mathrm{E} 2}$ & $\begin{array}{c}1056 \text { CABG recipients } \\
\text { (368 received ASA, } \\
688 \text { did not) }\end{array}$ & $\begin{array}{l}31 \% \text { experienced } \\
\text { in-hospital death }\end{array}$ & $\begin{array}{l}37 \% \text { experienced } \\
\text { in-hospital death }\end{array}$ & $\begin{array}{l}\text { ASA reduced the risk of in- } \\
\text { hospital death by } 45 \% \text { on } \\
\text { multivariate analysis }(\mathrm{OR}, \\
0.55 ; 95 \% \mathrm{CI}, 0.31-0.98 \\
P=.04)\end{array}$ \\
\hline Brown et $\mathrm{al}^{\mathrm{E} 3}$ & $\begin{array}{l}127 \text { CABG patients who } \\
\text { underwent CABG } \\
\text { followed by coronary } \\
\text { angiography at } 1 \text { year } \\
\text { (38 received ASA } \\
\text { alone, } 45 \text { received } \\
\text { ASA }+ \text { DP, } 44 \\
\text { received placebo) }\end{array}$ & $\begin{array}{l}12 \% \text { of grafts in ASA-only } \\
\text { recipients were occluded } \\
14 \% \text { of grafts in ASA } \\
\text { +DP recipients } \\
\text { were occluded }\end{array}$ & $\begin{array}{l}21 \% \text { of grafts } \\
\text { were occluded }\end{array}$ & $\begin{array}{l}\text { ASA alone reduced the odds of } \\
\text { occlusion at } 1 \text { year by } 53 \% \\
(\mathrm{OR}, 0.47 ; P=.04) \\
\text { ASA + DP reduced the odds of } \\
\text { occlusion at } 1 \text { year by } 50 \% \\
\text { (OR, } 0.50 ; P=.04)\end{array}$ \\
\hline $\begin{array}{c}\text { Gavaghan } \\
{\text { et } \mathrm{al}^{\mathrm{E} 4}}\end{array}$ & $\begin{array}{l}231 \text { patients who } \\
\text { underwent CABG } \\
\text { followed by coronary } \\
\text { angiography at } 1 \text { week } \\
\text { (126 received ASA, } \\
105 \text { did not) }\end{array}$ & $\begin{array}{l}4.0 \% \text { of grafts were } \\
\text { occluded }\end{array}$ & $\begin{array}{l}14.3 \% \text { of grafts were } \\
\text { occluded }(P=.003 \\
\text { vs those who received ASA })\end{array}$ & Not reported \\
\hline $\begin{array}{c}\text { Gavaghan } \\
\text { et }^{\mathrm{El}}{ }^{\mathrm{E}}\end{array}$ & $\begin{array}{l}219 \text { patients who } \\
\text { underwent } \\
\text { CABG followed by } \\
\text { coronary angiography } \\
\text { at } 1 \text { year (119 received } \\
\text { ASA, } 100 \text { did not) }\end{array}$ & $\begin{array}{l}11.9 \% \text { of grafts were } \\
\text { occluded }\end{array}$ & $\begin{array}{l}29.5 \% \text { of grafts were } \\
\text { occluded }(P<.002 \\
\text { vs those who received } \\
\text { ASA })\end{array}$ & Not reported \\
\hline Mangano $^{\mathrm{E} 5}$ & $\begin{array}{l}5022 \text { CABG recipients } \\
\text { (2999 received ASA, } \\
2023 \text { did not) }\end{array}$ & $\begin{array}{l}1.3 \% \text { experienced } \\
\text { in-hospital all-cause } \\
\text { death; } \\
1.1 \% \text { experienced } \\
\text { in-hospital cardiac } \\
\text { death; } \\
2.8 \% \text { experienced } \\
\text { in-hospital MI; } \\
1.3 \% \text { experienced } \\
\text { in-hospital stroke }\end{array}$ & $\begin{array}{l}4.0 \% \text { experienced } \\
\text { in-hospital all-cause } \\
\text { death; } \\
3.1 \% \text { experienced } \\
\text { in-hospital cardiac } \\
\text { death; } \\
5.4 \% \text { experienced } \\
\text { in-hospital MI; } \\
\text { 2.6\% experienced } \\
\text { in-hospital stroke }\end{array}$ & $\begin{array}{l}\text { ASA significantly reduced } \\
\text { the odds of in-hospital } \\
\text { all-cause mortality, } \\
\text { cardiac mortality, MI, } \\
\text { and stroke }(P \leq .01 \\
\text { for each) }\end{array}$ \\
\hline
\end{tabular}

$A S A$, Aspirin; $C A B G$, Coronary artery bypass grafting; $C I$, confidence interval; $D P$, dipyridamole; $M I$, myocardial infarction; $O R$, odds ratio. 
TABLE E2. Blood loss, blood product usage, and rates of reoperation for bleeding in the different eras of antiplatelet therapy

\begin{tabular}{|c|c|c|c|c|c|}
\hline Study & Blood lost (mL) & $\begin{array}{c}\text { Reoperation } \\
\text { for bleeding } \\
(n ; \%)\end{array}$ & $\begin{array}{c}\text { Blood } \\
\text { transfused } \\
(\text { U/patient; \%) } \\
\end{array}$ & $\begin{array}{c}\text { FFP } \\
\text { transfused } \\
(\text { U/patient; \%) } \\
\end{array}$ & $\begin{array}{c}\text { Platelets } \\
\text { transfused } \\
(\text { U/patient; \%) } \\
\end{array}$ \\
\hline \multicolumn{6}{|c|}{ A. Studies comparing aspirin vs no aspirin } \\
\hline Michelson et al ${ }^{\mathrm{E} 6}$ & $\begin{array}{l}\text { ASA: } 919 \pm 491^{\mathrm{a}, \mathrm{b}} \\
\text { No ASA: } 437 \pm 244^{\mathrm{a}}\end{array}$ & NR & $\begin{array}{l}\text { ASA: } 1.1 \pm 1.3^{\mathrm{a}} ; 55.6 \\
\text { No ASA: } 1.3 \pm 1.5^{\mathrm{a}} ; 68.8\end{array}$ & NR & NR \\
\hline Ferraris et al ${ }^{\mathrm{E} 7}$ & $\begin{array}{l}\text { ASA: } 1513 \pm 977^{\mathrm{a}, \mathrm{b}, \mathrm{c}} \\
\text { No ASA: } 916 \pm 482^{\mathrm{a}, \mathrm{c}}\end{array}$ & $\begin{array}{l}\text { ASA: } 2 ; 12.5 \\
\text { No ASA: } 0\end{array}$ & $\begin{array}{l}\text { ASA: } 4.4 \pm 3.5^{\mathrm{a}, \mathrm{b}} \\
\text { No ASA: } 1.8 \pm 1.3^{\mathrm{a}}\end{array}$ & $\begin{array}{l}\text { ASA: } 3.6 \pm 4.9^{\mathrm{a}, \mathrm{b}} \\
\text { No ASA: } 0.7 \pm 1.6^{\mathrm{a}}\end{array}$ & $\begin{array}{l}\text { ASA: } 1.3 \pm 1.3^{\mathrm{a}, \mathrm{b}} \\
\text { No ASA: } 0.16 \pm 0.38^{\mathrm{a}}\end{array}$ \\
\hline Taggart et $\mathrm{al}^{\mathrm{E} 8}$ & $\begin{array}{c}75 \text { ASA: } 1150 \\
(820,1380)^{\mathrm{b}, \mathrm{d}} \\
150 \text { ASA: } 1360 \\
(1020,1730)^{\mathrm{b}, \mathrm{d}} \\
300 \text { ASA: } 1100 \\
(810,1340)^{\mathrm{b}, \mathrm{d}} \\
\text { No ASA: } 870 \\
(790,960)^{\mathrm{d}}\end{array}$ & $\begin{array}{l}\text { ASA: } 7 ; 6.9 \\
\text { No ASA: } 6 ; 5.9\end{array}$ & $\begin{array}{l}75 \text { ASA: } 4.0(2.5,4.5)^{\mathrm{b}, \mathrm{e}} \\
\text { 150 ASA: } 4.0(2.5,5.0)^{\mathrm{b}, \mathrm{e}} \\
\text { 300 ASA: } 3.0(1.5,4.5)^{\mathrm{b}, \mathrm{e}} \\
\text { No ASA: } 2.0(1.5,2.5)^{\mathrm{e}}\end{array}$ & NR & NR \\
\hline Goldman et al ${ }^{\mathrm{E} 9}$ & $\begin{array}{l}325 \text { ASA: } 965^{\mathrm{b}, \mathrm{e}} \\
325 \text { ASA tid: } 1175^{\mathrm{b}, \mathrm{e}} \\
\text { ASA/DP: } 1000^{\mathrm{b}, \mathrm{e}} \\
\text { Sulf: } 775^{\mathrm{e}} \\
\text { Placebo: } 805^{\mathrm{e}}\end{array}$ & $\begin{array}{l}325 \text { ASA: NR; } 9.3^{\mathrm{f}} \\
325 \text { ASA tid: NR; } 6.3^{\mathrm{f}} \\
\text { ASA/DP: NR; } 3.9^{\mathrm{f}} \\
\text { Sulf: NR; } 2.1 \\
\text { Placebo: NR; } 1.3\end{array}$ & NR & NR & NR \\
\hline Dacey et al (a) ${ }^{\mathrm{E} 2}$ & $\begin{array}{l}\text { ASA: } 1318 \pm 2078^{\mathrm{a}} \\
\text { No ASA: } 1255 \pm 1498^{\mathrm{a}}\end{array}$ & $\begin{array}{l}\text { ASA: NR; } 32.9 \\
\text { No ASA: NR; } 33.0\end{array}$ & $\begin{array}{l}\text { ASA: } 3.0 \pm 4.1^{\mathrm{a}} \\
\text { No ASA: } 3.3 \pm 4.3^{\mathrm{a}}\end{array}$ & $\begin{array}{l}\text { ASA: } 3.4 \pm 5.7^{\mathrm{a}} \\
\text { No ASA: } 3.5 \pm 5.5^{\mathrm{a}}\end{array}$ & $\begin{array}{l}\text { ASA: } 3.6 \pm 8.3^{\mathrm{a}} \\
\text { No ASA: } 5.4 \pm 10.6^{\mathrm{a}}\end{array}$ \\
\hline Dacey et al $(b)^{\mathrm{E} 2}$ & $\begin{array}{l}\text { ASA: } 822 \pm 590^{\mathrm{a}} \\
\text { No ASA: } 799 \pm 774^{\mathrm{a}}\end{array}$ & $\begin{array}{l}\text { ASA: NR; } 7.1 \\
\text { No ASA: } 8.1\end{array}$ & $\begin{array}{l}\text { ASA: } 1.4 \pm 1.7^{\mathrm{a}} \\
\text { No ASA: } 1.4 \pm 2.0^{\mathrm{a}}\end{array}$ & $\begin{array}{l}\text { ASA: } 0.9 \pm 1.9^{\mathrm{a}} \\
\text { No ASA: } 1.1 \pm 2.9^{\mathrm{a}}\end{array}$ & $\begin{array}{l}\text { ASA: } 1.1 \pm 3.3^{\mathrm{a}} \\
\text { No ASA: } 1.2 \pm 4.0^{\mathrm{a}}\end{array}$ \\
\hline Kallis et $\mathrm{al}^{\mathrm{E} 10}$ & $\begin{array}{l}\text { ASA: } 1185^{\mathrm{b}, \mathrm{g}} \\
\text { Placebo: } 791^{\mathrm{g}}\end{array}$ & $\begin{array}{l}\text { ASA: } 4^{\mathrm{b}} ; 8 \\
\text { No ASA: } 0\end{array}$ & $\begin{array}{l}\text { ASA: } 3.1^{\mathrm{b}, \mathrm{g}} \\
\text { No ASA: } 2.1^{\mathrm{g}}\end{array}$ & $\begin{array}{l}\text { ASA: } 0.4^{\mathrm{b}, \mathrm{g}} \\
\text { No ASA: } 0^{\mathrm{g}}\end{array}$ & $\begin{array}{l}\text { ASA: } 0.5^{\mathrm{b}, \mathrm{g}} \\
\text { No ASA: } 0^{\mathrm{g}}\end{array}$ \\
\hline Tuman et al ${ }^{\mathrm{E} 11}$ & $\begin{array}{l}\text { ASA: } 627 \pm 388^{\mathrm{a}} \\
\text { No ASA: } 658 \pm 465^{\mathrm{a}}\end{array}$ & $\begin{array}{l}\text { ASA: } 4 ; 1.3 \\
\text { No ASA: } 0\end{array}$ & $\begin{array}{l}\text { ASA: } 1.4 \pm 1.9^{\mathrm{a}} \\
\text { No ASA: } 1.3 \pm 2.0^{\mathrm{a}}\end{array}$ & $\begin{array}{l}\text { ASA: } 0.5 \pm 1.3^{\mathrm{a}} \\
\text { No ASA: } 0.4 \pm 1.1^{\mathrm{a}}\end{array}$ & $\begin{array}{l}\text { ASA: } 0.5 \pm 1.2^{\mathrm{a}} \\
\text { No ASA: } 0.5 \pm 1.1^{\mathrm{a}}\end{array}$ \\
\hline Vuylsteke et $\mathrm{al}^{\mathrm{E} 12}$ & $\begin{array}{l}\text { ASA: } 685 \pm 327^{\mathrm{a}} \\
\text { No ASA: } 654 \pm 289^{\mathrm{a}}\end{array}$ & $\begin{array}{l}\text { ASA: } 1 ; 1.2 \\
\text { No ASA: } 1 ; 1.7\end{array}$ & $\begin{array}{l}\text { ASA: } 1.1 \pm 1.8^{\mathrm{a}} \\
\text { No ASA: } 1.5 \pm 3.2^{\mathrm{a}}\end{array}$ & $\begin{array}{l}\text { ASA: } 0.1 \pm 0.6^{\mathrm{a}} \\
\text { No ASA: } 0.2 \pm 0.2^{\mathrm{a}}\end{array}$ & $\begin{array}{l}\text { ASA: } 0.2 \pm 0.5^{\mathrm{a}} \\
\text { No ASA: } 0 \pm 0.8^{\mathrm{a}}\end{array}$ \\
\hline \multicolumn{6}{|c|}{ B. Studies comparing clopidogrel vs no clopidogrel } \\
\hline Hongo et al ${ }^{\mathrm{E} 13}$ & $\begin{array}{l}\text { CLO: } 1124 \pm 1119^{\mathrm{a}, \mathrm{b}} \\
\text { No CLO: } 775 \pm 727^{\mathrm{a}}\end{array}$ & $\begin{array}{l}\text { CLO: } 4 ; 6.8^{\mathrm{b}} \\
\text { No CLO: } 1 ; 0.6\end{array}$ & $\begin{array}{l}\text { CLO: } 2.51 \pm 2.41^{\mathrm{a}, \mathrm{b}} ; 79.7^{\mathrm{b}} \\
\text { No CLO: } 1.74 \pm 2.16^{\mathrm{a}} ; 50.8\end{array}$ & $\begin{array}{l}\text { CLO: } 0.68 \pm 1.66^{\mathrm{a}, \mathrm{b}} \\
\text { No CLO: } 0.24 \pm 0.85^{\mathrm{a}}\end{array}$ & $\begin{array}{l}\text { CLO: } 0.86 \pm 1.20^{\mathrm{a}, \mathrm{b}} ; 50.8^{\mathrm{b}} \\
\text { No CLO: } 0.24 \pm 0.60^{\mathrm{a}} ; 18.2\end{array}$ \\
\hline Englberger et al ${ }^{\mathrm{E} 14}$ & $\begin{array}{l}\text { CLO: } 1485 \pm 310^{\mathrm{a}, \mathrm{b}} \\
\text { No CLO: } 780 \pm 105^{\mathrm{a}}\end{array}$ & $\begin{array}{l}\text { CLO: } 8 ; 5.9^{\mathrm{b}} \\
\text { No CLO: } 5 ; 1.2\end{array}$ & $\begin{array}{l}\text { CLO: } 4.6 \pm 2.3^{\mathrm{a}, \mathrm{b}} ; 92^{\mathrm{b}} \\
\text { No CLO: } 1.5 \pm 2.2^{\mathrm{a}} ; 47\end{array}$ & $\begin{array}{l}\text { CLO: } 0.9 \pm 1.4^{\mathrm{a}} ; 42^{\mathrm{b}} \\
\text { No CLO: } 0.3 \pm 0.9^{\mathrm{a}} ; 13\end{array}$ & $\begin{array}{l}\text { CLO: } 2.6 \pm 1.1^{\mathrm{a}, \mathrm{b}} ; 71^{\mathrm{b}} \\
\text { No CLO: } 0.2 \pm 0.6^{\mathrm{a}} ; 9\end{array}$ \\
\hline Karabulut et $\mathrm{al}^{\mathrm{E} 15}$ & $\begin{array}{l}\text { CLO: } 719 \pm 265^{\mathrm{a}} \\
\text { No CLO: } 612 \pm 350^{\mathrm{a}}\end{array}$ & $\begin{array}{l}\text { CLO: } 0 \\
\text { No CLO: } 16 ; 1\end{array}$ & $\begin{array}{l}\text { CLO: } 0.5 \pm 0.9^{\mathrm{a}} \\
\text { No CLO: } 0.4 \pm 0.9^{\mathrm{a}}\end{array}$ & $\begin{array}{l}\text { CLO: } 1.1 \pm 1.2^{\mathrm{a}} \\
\text { No CLO: } 0.9 \pm 1.1^{\mathrm{a}}\end{array}$ & $\mathrm{NR}$ \\
\hline Kapetanakis et al ${ }^{\mathrm{E} 16}$ & $\begin{array}{l}\text { CLO: } 400(100-6000)^{\mathrm{h}} \\
\text { No CLO: } 400 \\
\quad(100-2000)^{\mathrm{h}}\end{array}$ & $\begin{array}{l}\text { CLO: } 24 ; 5.8^{\mathrm{b}} \\
\text { No CLO: } 25 ; 1.3\end{array}$ & $\begin{array}{l}\text { CLO: } 2.0 \\
\quad(1.0-8.0)^{\mathrm{b}, \mathrm{h}} ; 30.4^{\mathrm{b}} \\
\text { No CLO: } 2.0 \\
\quad(1.0-7.0)^{\mathrm{h}} ; 22.1\end{array}$ & $\begin{array}{l}\text { CLO: } 1.5(0.8-3.1)^{\mathrm{h}} ; 3.9^{\mathrm{b}} \\
\text { No CLO: } 1.9(0.1-3.8)^{\mathrm{h}} ; 1.6\end{array}$ & $\begin{array}{l}\text { CLO: } 0.7(0.6-1.2)^{\mathrm{h}} ; 5.1^{\mathrm{b}} \\
\text { No CLO: } 0.7(0.2-1.3)^{\mathrm{h}} ; 1.8\end{array}$ \\
\hline Mehta et al (a) $)^{\mathrm{E} 17}$ & NR & NR & $\begin{array}{l}\text { CLO: } 2(0,4)^{\mathrm{b}, \mathrm{i}} \\
\text { No CLO: } 1(0,3)^{\mathrm{i}}\end{array}$ & NR & $\begin{array}{l}\text { CLO: NR; } 33.7^{\mathrm{b}} \\
\text { No CLO: NR; } 20.3\end{array}$ \\
\hline Mehta et al $(b)^{\mathrm{E} 17}$ & NR & NR & $\begin{array}{l}\text { CLO: } 2(0,4)^{\mathrm{i}} \\
\text { No CLO: } 2(0,4)^{\mathrm{i}}\end{array}$ & NR & $\begin{array}{l}\text { CLO: NR; } 17.7 \\
\text { No CLO: NR; } 15.0\end{array}$ \\
\hline Berger et $\mathrm{al}^{\mathrm{E} 18}$ & NR & $\begin{array}{l}\text { CLO: } 14 ; 4.7^{\mathrm{b}} \\
\text { No CLO: } 4 ; 1.3\end{array}$ & NR & NR & NR \\
\hline Kim et $\mathrm{al}^{\mathrm{E} 19}$ & NR & $\begin{array}{l}\text { CLO: } 11 ; 3.3 \\
\text { No CLO: } 116 ; 2.6\end{array}$ & $\begin{array}{l}\text { CLO: NR; } 70.2 \\
\text { No CLO: NR; } 68.2\end{array}$ & NR & NR \\
\hline \multicolumn{6}{|c|}{$\begin{array}{l}\text { C. Studies comparing other antiplatelet regimens (combination vs monotherapy, antiplatelet vs no antiplatelet, different time frames of } \\
\text { antiplatelet exposure) }\end{array}$} \\
\hline $\begin{array}{l}\text { Yende and } \\
\text { Wunderink }\end{array}$ & NR & $\begin{array}{l}\text { ASA + CLO: } \\
\quad 5 ; 10.4^{\mathrm{j}} \\
\text { ASA: } 3 ; 2.3^{\mathrm{j}} \\
\text { Neither: } 0^{\mathrm{j}}\end{array}$ & $\begin{array}{l}\text { ASA + CLO: } 2.9^{\mathrm{g}, \mathrm{k}} ; 70.8^{\mathrm{k}} \\
\text { ASA: } 1.8^{\mathrm{g}} ; 53.9 \\
\text { Neither: } 1.1^{\mathrm{g}} ; 46.9\end{array}$ & $\begin{array}{l}\text { ASA + CLO: } 1.2^{\mathrm{g}} ; 31.3 \\
\text { ASA: } 0.7^{\mathrm{g}} ; 23.9 \\
\text { Neither: } 0.5^{\mathrm{g}} ; 17.2\end{array}$ & $\begin{array}{l}\text { ASA + CLO: } 4.6^{\mathrm{g}, \mathrm{k}} \\
\text { ASA: } 2.1^{\mathrm{g}} \\
\text { Neither: } 0.7^{\mathrm{g}}\end{array}$ \\
\hline Ray et $\mathrm{al}^{\mathrm{E} 21}$ & NR & NR & $\begin{array}{l}\text { ASA +CLO: } 2.4 \pm 2.3^{\mathrm{a}, \mathrm{j}} \\
\text { ASA: } 1.9 \pm 2.4^{\mathrm{a}, \mathrm{j}} \\
\text { CLO: } 2.9 \pm 3.4^{\mathrm{a}, \mathrm{j}} \\
\text { Neither: } 1.4 \pm 2.0^{\mathrm{a}}\end{array}$ & $\begin{array}{l}\text { ASA + CLO: } 3.1 \pm 5.4^{\mathrm{a}, \mathrm{j}} \\
\text { ASA: } 2.3 \pm 4.4^{\mathrm{a}, \mathrm{j}} \\
\text { CLO: } 2.5 \pm 5.4^{\mathrm{a}, \mathrm{j}} \\
\text { Neither: } 1.6 \pm 2.9^{\mathrm{a}}\end{array}$ & $\begin{array}{l}\text { ASA +CLO: } 3.7 \pm 5.2^{\mathrm{a}, \mathrm{j}} \\
\text { ASA: } 1.3 \pm 3.1^{\mathrm{a}, \mathrm{j}} \\
\text { CLO: } 3.6 \pm 6.0^{\mathrm{a}, \mathrm{j}} \\
\text { Neither: } 1.0 \pm 3.7^{\mathrm{a}, \mathrm{j}}\end{array}$ \\
\hline
\end{tabular}


TABLE E2. Continued

\begin{tabular}{|c|c|c|c|c|c|}
\hline Study & Blood lost (mL) & $\begin{array}{l}\text { Reoperation } \\
\text { for bleeding } \\
\quad(\mathbf{n} ; \%)\end{array}$ & $\begin{array}{c}\text { Blood } \\
\text { transfused } \\
(\mathrm{U} / \text { patient; \%) }\end{array}$ & $\begin{array}{c}\text { FFP } \\
\text { transfused } \\
(\mathrm{U} / \text { patient; } \%)\end{array}$ & $\begin{array}{c}\text { Platelets } \\
\text { transfused } \\
\text { (U/patient; \%) }\end{array}$ \\
\hline $\begin{array}{l}\text { Leong } \\
\quad \text { et al (a) }\end{array}$ & $\begin{array}{l}\text { ASA + CLO: } 503(225- \\
\quad \text { 1230 })^{\mathrm{h}} \\
\text { ASA: } 570(100-3675)^{\mathrm{h}} \\
\text { CLO: } 573(140-1365)^{\mathrm{h}} \\
\text { Neither: } 503(225-1230)^{\mathrm{h}}\end{array}$ & $\begin{array}{l}\text { ASA + CLO: } 0 \\
\text { ASA: } 0 \\
\text { CLO: } 0 \\
\text { Neither: } 0\end{array}$ & $\begin{array}{l}\text { ASA + CLO: } 0(0-0)^{\mathrm{h}} \\
\text { ASA: } 0(0-4)^{\mathrm{h}} \\
\text { CLO: } 0(0-2)^{\mathrm{h}} \\
\text { Neither: } 0(0-10)^{\mathrm{h}}\end{array}$ & $\begin{array}{l}\text { ASA + CLO: } 0(0-0)^{\mathrm{h}} \\
\text { ASA: } 0(0-2)^{\mathrm{h}} \\
\text { CLO: } 0(0-0)^{\mathrm{h}} \\
\text { Neither: } 0(0-0)^{\mathrm{h}}\end{array}$ & $\begin{array}{l}\text { ASA + CLO: } 0(0-0)^{\mathrm{h}} \\
\text { ASA: } 0(0-6)^{\mathrm{h}} \\
\text { CLO: } 0(0-0)^{\mathrm{h}} \\
\text { Neither: } 0(0-0)^{\mathrm{h}}\end{array}$ \\
\hline $\begin{array}{l}\text { Leong } \\
\text { et al (b) }{ }^{\mathrm{E} 22}\end{array}$ & $\begin{array}{l}\text { ASA + CLO: } 803 \\
\quad(300-3780)^{\mathrm{h}, \mathrm{k}} \\
\text { ASA: } 620(58-4500)^{\mathrm{h}} \\
\text { CLO: } 585(200-1755)^{\mathrm{h}} \\
\text { Neither: } 603(50-2908)^{\mathrm{h}}\end{array}$ & $\begin{array}{l}\text { ASA + CLO: } 27 ; 3.4 \\
\text { ASA: } 1 ; 1.5 \\
\text { CLO: } 0 \\
\text { Neither: } 1 ; 0.5\end{array}$ & $\begin{array}{l}\text { ASA + CLO: } 1(1-30)^{\mathrm{h}} \\
\text { ASA: } 1(0-12)^{\mathrm{h}} \\
\text { CLO: } 1(0-10)^{\mathrm{h}} \\
\text { Neither: } 1(0-21)^{\mathrm{h}}\end{array}$ & $\begin{array}{l}\text { ASA + CLO: } 0(0-4)^{\mathrm{h}} \\
\text { ASA: } 0(0-11)^{\mathrm{h}} \\
\text { CLO: } 0(0-2)^{\mathrm{h}} \\
\text { Neither: } 0(0-3)^{\mathrm{h}}\end{array}$ & $\begin{array}{l}\text { ASA + CLO: } 0(0-5)^{\mathrm{h}} \\
\text { ASA: } 0(0-6)^{\mathrm{h}} \\
\text { CLO: } 0(0-1)^{\mathrm{h}} \\
\text { Neither: } 0(0-7)^{\mathrm{h}}\end{array}$ \\
\hline $\begin{array}{l}\text { Nurozler } \\
\text { et al }^{\mathrm{E} 23}\end{array}$ & $\begin{array}{l}\text { ASA + CLO: } \\
\quad 1180 \pm 280^{\mathrm{a}, \mathrm{j}} \\
\text { ASA: } 660 \pm 110^{\mathrm{a}, \mathrm{j}} \\
\text { CLO: } 1210 \pm 260^{\mathrm{a}, \mathrm{j}} \\
\text { Neither: } 625 \pm 90^{\mathrm{a}}\end{array}$ & $\begin{array}{l}\text { ASA + CLO: } 3 ; 6.1^{\mathrm{j}} \\
\text { ASA: } 1 ; 1.4^{\mathrm{j}} \\
\text { CLO: } 2 ; 7.1^{\mathrm{j}} \\
\text { Neither: } 0\end{array}$ & $\begin{array}{l}\text { ASA +CLO: } 3.9 \pm 0.8^{\mathrm{a}, \mathrm{j}} \\
\text { ASA: } 1.4 \pm 0.3^{\mathrm{a}, \mathrm{j}} \\
\text { CLO: } 3.7 \pm 1.0^{\mathrm{a}, \mathrm{j}} \\
\text { Neither: } 1.2 \pm 0.4^{\mathrm{a}}\end{array}$ & $\begin{array}{l}\text { ASA+CLO: } 1.4 \pm 0.6^{\mathrm{a}, \mathrm{j}} \\
\text { ASA: } 0.7 \pm 0.4^{\mathrm{a}, \mathrm{j}} \\
\text { CLO: } 1.3 \pm 0.7^{\mathrm{a}, \mathrm{j}} \\
\text { Neither: } 0.4 \pm 0.3^{\mathrm{a}}\end{array}$ & $\begin{array}{l}\text { ASA +CLO: } 2.0 \pm 1.2^{\mathrm{a}} \\
\text { ASA: } 0.2 \pm 0.5^{\mathrm{a}} \\
\text { CLO: } 2.2 \pm 1.1^{\mathrm{a}} \\
\text { Neither: } 0.3 \pm 0.4^{\mathrm{a}}\end{array}$ \\
\hline $\begin{array}{l}\text { Hekmat } \\
\text { et al }^{\mathrm{E} 24}\end{array}$ & $\begin{array}{l}\text { APT: } 1123 \pm 573^{\mathrm{a}, \mathrm{b}} \\
\text { No APT: } 874 \pm 351^{\mathrm{a}}\end{array}$ & $\begin{array}{l}\text { APT: } 4 ; 2.8 \\
\text { No APT: } 5 ; 3.4\end{array}$ & $\begin{array}{l}\text { APT: } 2.6 \pm 2.5^{\mathrm{a}, \mathrm{b}} \\
\text { No APT: } 1.6 \pm 1.8^{\mathrm{a}}\end{array}$ & $\begin{array}{l}\text { APT: } 2.0 \pm 2.2^{\mathrm{a}, \mathrm{b}} \\
\text { No APT: } 1.3 \pm 2.0^{\mathrm{a}}\end{array}$ & $\begin{array}{l}\text { APT: } 1.2 \pm 1.8^{\mathrm{a}, \mathrm{b}} \\
\text { No APT: } 0.2 \pm 0.8^{\mathrm{a}}\end{array}$ \\
\hline Shim et al ${ }^{\mathrm{E} 25}$ & $\begin{array}{l}\text { APT } \geq 6 \text { d: } 265 \pm 146^{\mathrm{a}, 1} \\
\text { APT } 3-5 \mathrm{~d}: 330 \pm 191^{\mathrm{a}, 1} \\
\text { APT } \leq 2 \mathrm{~d}: 323 \pm 187^{\mathrm{a}, 1} \\
\text { APT } \geq 6 \mathrm{~d}: 756 \pm 408^{\mathrm{a}, \mathrm{m}} \\
\text { APT } 3-5 \mathrm{~d}: 729 \pm 485^{\mathrm{a}, \mathrm{m}} \\
\text { APT } \leq 2 \mathrm{~d}: 627 \pm 257^{\mathrm{a}, \mathrm{m}}\end{array}$ & NR & $\begin{array}{l}\text { APT } \geq 6 \text { d: } 1.1 \pm 1.8^{\mathrm{a}} ; 39 \\
\text { APT } 3-5 \mathrm{~d}: 1.9 \pm 0.8^{\mathrm{a}} ; 48 \\
\text { APT } \leq 2 \mathrm{~d}: 1.6 \pm 1.9^{\mathrm{a}} ; 26\end{array}$ & $\begin{array}{l}\text { APT } \geq 6 \text { d: NR; } 11 \\
\text { APT 3-5 d: NR; } 8 \\
\text { APT } \leq 2 \text { d: NR; } 5\end{array}$ & $\begin{array}{l}\text { APT } \geq 6 \text { d: NR; } 0 \\
\text { APT 3-5 d: NR; } 2 \\
\text { APT } \leq 2 \text { d: NR; } 5\end{array}$ \\
\hline
\end{tabular}


TABLE E3. Details of studies reporting correlations between aspirin and clopidogrel therapy and blood loss, blood product usage and reoperation for bleeding

\begin{tabular}{|c|c|c|c|}
\hline Study reference & Description & Total $(\mathbf{N})$ & Antiplatelet regimen (n) \\
\hline $\begin{array}{l}\text { Berger et al. J Am Coll Cardiol. } \\
\text { 2008;52:1693-701 }\end{array}$ & $\begin{array}{l}\text { Retrospective, multicenter study of } \\
\text { patients who underwent CABG } \\
\text { within } 7 \mathrm{~d} \text { of ACS }\end{array}$ & 596 & $\begin{array}{l}\text { CLO } \leq 5 \text { d before CABG }(298)^{\mathrm{a}} \\
\text { No CLO (298) }\end{array}$ \\
\hline $\begin{array}{l}\text { Dacey et al. (a) Ann Thorac Surg. } \\
\text { 2000;70:1986-90 }{ }^{\mathrm{E} 2}\end{array}$ & $\begin{array}{l}\text { Retrospective, multicenter, case- } \\
\text { control study of patients who died } \\
\text { after CABG }\end{array}$ & 368 & $\begin{array}{l}\text { ASA (114) } \\
\text { No ASA (254) }\end{array}$ \\
\hline $\begin{array}{l}\text { Dacey et al. (b) Ann Thorac Surg. } \\
\text { 2000;70:1986-90 } \mathrm{E2}\end{array}$ & $\begin{array}{l}\text { Retrospective, multicenter, case- } \\
\text { control study of patients who } \\
\text { survived CABG }\end{array}$ & 688 & $\begin{array}{l}\text { ASA (254) } \\
\text { No ASA (434) }\end{array}$ \\
\hline $\begin{array}{l}\text { Englberger et al. Eur J Cardiothorac } \\
\quad \text { Surg. 2004;26:96-101 }\end{array}$ & $\begin{array}{l}\text { Prospective, single-site study of } \\
\text { patients undergoing consecutive } \\
\text { CABG procedures }\end{array}$ & 505 & $\begin{array}{l}\text { CLO } \leq 72 \mathrm{~h} \text { before CABG }(136)^{\mathrm{b}} \\
\text { No CLO }(369)\end{array}$ \\
\hline $\begin{array}{l}\text { Ferraris et al. Ann Thorac Surg. } \\
\qquad 1988 ; 45: 71-4^{\mathrm{E} 7}\end{array}$ & $\begin{array}{l}\text { Nonblinded, single-site randomized } \\
\text { study of patients undergoing } \\
\text { CABG procedures }\end{array}$ & 34 & $\begin{array}{l}\text { ASA the day before surgery (16) } \\
\text { No ASA (18) }\end{array}$ \\
\hline $\begin{array}{l}\text { Goldman et al. Circulation. } \\
\text { 1988;77:1324-32 }\end{array}$ & $\begin{array}{l}\text { Randomized, multicenter study of } \\
\text { patients undergoing CABG } \\
\text { procedures }\end{array}$ & 772 & $\begin{array}{l}325 \mathrm{mg} / \mathrm{d} \text { ASA }<48 \mathrm{~h} \text { of surgery }(154) \\
325 \mathrm{mg} \text { tid ASA }(155) \\
325 \mathrm{mg} \text { ASA }+75 \mathrm{mg} \text { DP tid }(162) \\
267 \mathrm{mg} \text { tid sulf }(148) \\
\text { Placebo (153) }\end{array}$ \\
\hline $\begin{array}{l}\text { Hekmat et al. Curr Med Res Opin. } \\
\text { 2004;20:1429-35 }\end{array}$ & $\begin{array}{l}\text { Retrospective, single-site study of } \\
\text { patients undergoing CABG } \\
\text { procedures }\end{array}$ & 290 & $\begin{array}{l}\text { APT } \leq 5 \text { d before CABG (145) } \\
\text { No APT (145) }\end{array}$ \\
\hline $\begin{array}{l}\text { Hongo et al. J Am Coll Cardiol. } \\
\qquad 2002 ; 40: 231-7^{13}\end{array}$ & $\begin{array}{l}\text { Prospective, single-site study of } \\
\text { patients undergoing CABG } \\
\text { procedures }\end{array}$ & 224 & $\begin{array}{l}\text { CLO } \leq 7 \text { d before CABG }(59)^{c} \\
\text { No CLO }(165)\end{array}$ \\
\hline $\begin{array}{l}\text { Kallis et al. Eur J Cardiothorac Surg. } \\
\qquad 1994 ; 8: 404-9^{\text {E10 }}\end{array}$ & $\begin{array}{l}\text { Randomized, single-site study of } \\
\text { patients undergoing elective } \\
\text { CABG procedures }\end{array}$ & 100 & $\begin{array}{l}300 \mathrm{mg} / \mathrm{d} \text { ASA until time of surgery (50) } \\
\text { Placebo (50) }\end{array}$ \\
\hline $\begin{array}{l}\text { Kapetanakis et al. Eur Heart } J \text {. } \\
2005 ; 26: 576-83^{\text {E16 }}\end{array}$ & $\begin{array}{l}\text { Retrospective, single-site study of } \\
\text { patients undergoing CABG } \\
\text { procedures }\end{array}$ & 2359 & $\begin{array}{l}\text { CLO } \leq 7 \text { d before CABG }(415)^{\mathrm{a}} \\
\text { No CLO (1944) }\end{array}$ \\
\hline $\begin{array}{l}\text { Karabulut et al. Eur J Cardiothorac } \\
\text { Surg. 2004;25:419-23 }\end{array}$ & $\begin{array}{l}\text { Retrospective, single-site study of } \\
\text { CABG procedures }\end{array}$ & 1628 & $\begin{array}{l}\text { CLO } \leq 2 \text { d before CABG }(48)^{d} \\
\text { No CLO }(1580)\end{array}$ \\
\hline $\begin{array}{r}\text { Kim et al. Am Heart J. } \\
2008 ; 156: 886-92^{\text {E19 }}\end{array}$ & $\begin{array}{l}\text { Retrospective, single-site study of } \\
\text { CABG procedures }\end{array}$ & 4794 & $\begin{array}{l}\text { CLO } \leq 5 \text { d before CABG }(332)^{\mathrm{e}} \\
\text { No CLO }(4462)\end{array}$ \\
\hline $\begin{array}{l}\text { Leong et al. (a) Ann Thorac Surg. } \\
\text { 2005;80:928-33 }\end{array}$ & $\begin{array}{l}\text { Retrospective, single-site study of } \\
\text { patients undergoing OPCABG } \\
\text { procedures }\end{array}$ & 109 & $\begin{array}{l}\text { ASA }+ \text { CLO } \leq 7 \text { d before CABG }(2) \\
\text { ASA alone (75) } \\
\text { CLO alone (6) } \\
\text { Neither drug (26) }\end{array}$ \\
\hline $\begin{array}{l}\text { Leong et al. (b) Ann Thorac Surg. } \\
\text { 2005;80:928-33 }\end{array}$ & $\begin{array}{l}\text { Retrospective, single-site study of } \\
\text { patients undergoing CABG CPB } \\
\text { procedures }\end{array}$ & 810 & $\begin{array}{l}\text { ASA }+ \text { CLO } \leq 7 \text { d before CABG }(59) \\
\text { ASA alone }(523) \\
\text { CLO alone (18) } \\
\text { Neither drug (210) }\end{array}$ \\
\hline $\begin{array}{l}\text { Mehta et al. (a) J Am Coll Cardiol. } \\
\text { 2006;48:281-6 }\end{array}$ & $\begin{array}{l}\text { Retrospective, multisite study of } \\
\text { patients undergoing CABG } \\
\text { procedures performed } \leq 5 \mathrm{~d} \text { of } \\
\text { symptom onset }\end{array}$ & 2565 & $\begin{array}{l}\text { CLO }(739)^{\mathrm{f}} \\
\text { No CLO }(1826)\end{array}$ \\
\hline $\begin{array}{l}\text { Mehta et al. (b) J Am Coll Cardiol. } \\
\text { 2006;48:281-6 }\end{array}$ & $\begin{array}{l}\text { Retrospective, multisite study of } \\
\text { patients undergoing CABG } \\
\text { procedures performed }>5 \mathrm{~d} \text { after } \\
\text { symptom }\end{array}$ & 293 & $\begin{array}{l}\text { CLO }>5 \text { d before CABG }(113)^{\mathrm{g}} \\
\text { No CLO }(180)\end{array}$ \\
\hline
\end{tabular}


TABLE E3. Continued

\begin{tabular}{|c|c|c|c|}
\hline Study reference & Description & Total $(\mathbf{N})$ & Antiplatelet regimen (n) \\
\hline $\begin{array}{l}\text { Michelson et al. J Thorac } \\
\text { Cardiovasc Surg. 1978;76: } \\
694-7^{\mathrm{E} 6}\end{array}$ & $\begin{array}{l}\text { Retrospective, single-site study of } \\
\text { patients undergoing CABG } \\
\text { procedures }\end{array}$ & 25 & $\begin{array}{l}\text { ASA in the week before surgery (9) } \\
\text { No ASA (16) }\end{array}$ \\
\hline $\begin{array}{l}\text { Nurozler et al. Interact Cardiovasc } \\
\text { Thorac Surg. 2005;4:546-9 }{ }^{\mathrm{E} 23}\end{array}$ & $\begin{array}{l}\text { Retrospective, single-site study of } \\
\text { patients undergoing CABG } \\
\text { procedures }\end{array}$ & 182 & $\begin{array}{l}\text { ASA }+ \text { CLO } \leq 3 \text { d before CABG (49) } \\
\text { ASA alone (68) } \\
\text { CLO alone (28) } \\
\text { Neither drug (37) }\end{array}$ \\
\hline $\begin{array}{l}\text { Ray et al. BMC Cardiovasc Disord. } \\
\qquad 2003 ; 3: 3^{\mathrm{E} 21}\end{array}$ & $\begin{array}{l}\text { Retrospective, multisite study of } \\
\text { patients undergoing CABG } \\
\text { procedures }\end{array}$ & 659 & $\begin{array}{l}\text { ASA }+ \text { CLO } \leq 7 \mathrm{~d} \text { before CABG }(46) \\
\text { ASA alone }(105) \\
\text { CLO alone (11) } \\
\text { Neither drug (497) }\end{array}$ \\
\hline $\begin{array}{l}\text { Shim et al. } J \text { Thorac Cardiovasc } \\
\text { Surg. 2007;134:59-64 }\end{array}$ & $\begin{array}{l}\text { Prospective, single-site study of } \\
\text { patients undergoing OPCABG } \\
\text { procedures }\end{array}$ & 106 & $\begin{array}{l}\text { ASA + CLO } \geq 6 \mathrm{~d} \text { before CABG (35) } \\
\text { ASA + CLO } 3-5 \mathrm{~d} \text { before CABG (51) } \\
\text { ASA + CLO } \leq 2 \mathrm{~d} \text { before CABG (20) }\end{array}$ \\
\hline $\begin{array}{l}\text { Taggart et al. Ann Thorac Surg. } \\
\text { 1990;50:424-8 } 8^{\mathrm{E} 8}\end{array}$ & $\begin{array}{l}\text { Prospective, single-site study of } \\
\text { consecutive patients undergoing } \\
\text { CABG procedures }\end{array}$ & 202 & $\begin{array}{l}75 \mathrm{mg} / \mathrm{d} \text { ASA at time of surgery (44) } \\
150 \mathrm{mg} / \mathrm{d} \text { ASA (28) } \\
300 \mathrm{mg} / \mathrm{d} \text { ASA (29) } \\
\text { No ASA (101) }\end{array}$ \\
\hline $\begin{array}{l}\text { Tuman et al. Anesth Analg. } \\
\text { 1996;83:1178-84 }\end{array}$ & $\begin{array}{l}\text { Prospective, single-site study of } \\
\text { patients undergoing elective } \\
\text { CABG procedures }\end{array}$ & 317 & $\begin{array}{l}\text { ASA } \leq 7 \text { d before surgery (215) } \\
\text { No ASA (102) }\end{array}$ \\
\hline $\begin{array}{l}\text { Vuylsteke et al. J Cardiothorac Vasc } \\
\text { Anesth. 1997;11:831-4 }\end{array}$ & $\begin{array}{l}\text { Prospective, single-site study of } \\
\text { patients undergoing CABG } \\
\text { procedures }\end{array}$ & 144 & $\begin{array}{l}\text { ASA } \leq 7 \text { d before CABG ( } 86) \\
\text { No ASA ( } 58)\end{array}$ \\
\hline $\begin{array}{l}\text { Yende and Wunderink. Crit Care } \\
\text { Med. 2001;29:2271-5 }\end{array}$ & $\begin{array}{l}\text { Prospective, single-site study of } \\
\text { patients undergoing CABG } \\
\text { procedures }\end{array}$ & 245 & $\begin{array}{l}\text { ASA }+ \text { CLO } \leq 5 \text { d before CABG (48) } \\
\text { ASA alone (130) } \\
\text { Neither drug (64) }\end{array}$ \\
\hline
\end{tabular}

Studies are listed alphabetically. See Table E2 for study findings. $A C S$, acute coronary syndrome; $A P T$, Antiplatelet; $A S A$, aspirin; $C A B G$, coronary artery bypass grafting; $C L O$, clopidogrel; $C P B$, cardiopulmonary bypass; $d$, day(s); $D P$, dipyridamole; $h$, hour(s); $N R$, not reported; $O P C A B G$, off-pump coronary artery bypass grafting; sulf, sulfinpyrazone; tid, three times daily. ${ }^{a} \mathrm{All}$ CLO and no CLO recipients received ASA; ${ }^{\mathrm{b}}$ The exact percentages of patients taking ASA not reported, although text indicates the use was comparable between CLO and no CLO groups; ${ }^{c} 86.4 \%$ of CLO recipients received ASA vs $47.3 \%$ of no CLO recipients $(P<.001) ;{ }^{\mathrm{d}} 56 \%$ of CLO recipients and $65 \%$ of no CLO recipients received ASA $\left(P=\right.$ not significant); ${ }^{\mathrm{e}} 76.6 \%$ of CLO recipients and $79.2 \%$ of no CLO recipients received ASA ( $P$ not provided); ${ }^{\mathrm{f}} 96.6 \%$ of CLO recipients and $94.9 \%$ of no CLO recipients received ASA ( $P=$ not significant); ${ }^{\mathrm{g}} 95.5 \%$ of CLO recipients and $90.5 \%$ of no CLO recipients received ASA $(P=$ not significant). 
TABLE E4. Large clinical trials assessing the efficacy and safety of dual antiplatelet therapy in acute coronary syndromes

\begin{tabular}{|c|c|c|c|c|c|}
\hline Study & Patients & $\begin{array}{c}\text { Regimen }^{\mathrm{a}} \text { and } \\
\text { follow-up period }\end{array}$ & $\begin{array}{l}\text { Primary } \\
\text { end point }\end{array}$ & $\begin{array}{c}\text { Absolute } \\
\text { risk reduction }\end{array}$ & Bleeding \\
\hline CURE $^{\mathrm{E} 26}$ & 12,562 ACS patients & $\begin{array}{l}\text { CLO ( } 300 \mathrm{mg} \mathrm{LD} \text {, } \\
75 \mathrm{mg} / \mathrm{d} \mathrm{MD}) \mathrm{vs} \\
\text { placebo for an average } \\
\text { of } 9 \mathrm{mo}\end{array}$ & $\begin{array}{l}\text { CV death, nonfatal } \\
\text { MI, or stroke }\end{array}$ & $\begin{array}{l}2.1 \%(9.3 \% \text { vs } \\
\quad 11.4 \% ; P<.001)\end{array}$ & $\begin{array}{l}3.7 \% \text { vs } 2.7 \%^{\mathrm{b}} \\
\quad P=.001\end{array}$ \\
\hline PCI-CURE ${ }^{\mathrm{E} 27}$ & $\begin{array}{l}2658 \text { ACS patients } \\
\text { who underwent PCI }\end{array}$ & $\begin{array}{l}\text { CLO (300 mg LD, } \\
75 \mathrm{mg} / \mathrm{d} \mathrm{MD}) \mathrm{vs} \\
\text { placebo }\end{array}$ & $\begin{array}{l}\text { CV death, MI, or urgent } \\
\text { revascularization } \\
\text { within } 30 \mathrm{~d} \text { of PCI }\end{array}$ & $\begin{array}{l}1.9 \%(4.5 \% \text { vs } 6.4 \% ; \\
\quad P=.03)\end{array}$ & $\begin{array}{l}1.6 \% \text { vs } 1.4 \%{ }^{\mathrm{b}} \\
\quad P=.69\end{array}$ \\
\hline CABG-CURE ${ }^{\mathrm{E} 28}$ & $\begin{array}{l}2072 \text { ACS patients } \\
\text { who underwent } \\
\text { CABG }\end{array}$ & $\begin{array}{l}\text { CLO (300 mg LD, } \\
75 \mathrm{mg} / \mathrm{d} \mathrm{MD}) \mathrm{vs} \\
\text { placebo }\end{array}$ & $\begin{array}{l}\text { CV death, MI, } \\
\text { or stroke }\end{array}$ & $\begin{array}{l}1.7 \%(14.5 \% \text { vs } \\
16.2 \%)^{\mathrm{c}}\end{array}$ & $\begin{array}{l}9.6 \% \text { vs } 7.5 \%{ }^{\mathrm{b}} \\
\quad P=.095\end{array}$ \\
\hline CREDO $^{\mathrm{E} 29}$ & $\begin{array}{l}2116 \text { patients } \\
\text { undergoing elective } \\
\text { PCI }\end{array}$ & $\begin{array}{l}\text { CLO (300 mg LD, } \\
75 \mathrm{mg} / \mathrm{d} \mathrm{MD}) \mathrm{vs} \\
\text { placebo for } 12 \mathrm{mo}\end{array}$ & $\begin{array}{l}\text { All-cause death, } \\
\text { MI, or stroke }\end{array}$ & $\begin{array}{l}3.0 \%(8.5 \% \text { vs } \\
\quad 11.5 \% ; P=.02)\end{array}$ & $\begin{array}{l}8.8 \% \text { vs } 6.7 \% \text { d } \\
\quad P=.07\end{array}$ \\
\hline COMMIT $^{\mathrm{E} 30}$ & $\begin{array}{l}\text { 45,852 STEMI } \\
\text { patients }\end{array}$ & $\begin{array}{l}\text { CLO }(75 \mathrm{mg} / \mathrm{d} \mathrm{MD}) \\
\text { vs placebo } \\
\text { for an average } \\
\text { of } 15 \text { days }\end{array}$ & $\begin{array}{l}\text { All-cause death, } \\
\text { reinfarction, or stroke } \\
\text { before hospital } \\
\text { discharge } \\
\text { (up to } 4 \mathrm{wk} \text { ) }\end{array}$ & $\begin{array}{l}0.9 \% \text { for combined } \\
\text { end point } \\
(9.2 \% \text { vs } 10.1 \% \\
P=.002) \\
0.6 \% \text { for death } \\
\text { alone } \\
(8.1 \% \text { vs } 7.5 \% \\
P=.03)\end{array}$ & $\begin{array}{c}0.58 \% \text { vs } 0.55 \% \text {; } \\
\quad P=.59\end{array}$ \\
\hline CLARITY $^{\mathrm{E} 31}$ & $\begin{array}{l}3491 \text { STEMI } \\
\text { patients }\end{array}$ & $\begin{array}{l}\text { CLO (300 mg LD, } \\
75 \mathrm{mg} / \mathrm{d} \mathrm{MD}) \mathrm{vs} \\
\text { placebo for } 30 \mathrm{~d}\end{array}$ & $\begin{array}{l}\text { Composite of an } \\
\text { occluded } \\
\text { infarct-related artery } \\
\text { on angiography } \\
\text { or all-cause } \\
\text { death or recurrent MI } \\
\text { before angiography }\end{array}$ & $\begin{array}{l}6.7 \%(15.0 \% \text { vs } 21.7 \% \\
\quad P<.001)\end{array}$ & $\begin{array}{l}1.3 \% \text { vs } 1.1 \%{ }^{\mathrm{d}} ; \\
\quad P=.64\end{array}$ \\
\hline PCI-CLARITY ${ }^{\mathrm{E} 32}$ & $\begin{array}{l}1863 \text { STEMI } \\
\text { patients who } \\
\text { underwent PCI }\end{array}$ & $\begin{array}{l}\text { CLO (300 mg LD, } \\
75 \mathrm{mg} / \mathrm{d} \mathrm{MD}) \mathrm{vs} \\
\text { placebo for } 30 \mathrm{~d}\end{array}$ & $\begin{array}{l}\text { CV death, } \\
\text { nonfatal MI, or stroke } \\
\text { from PCI to } 30 \mathrm{~d}\end{array}$ & $\begin{array}{l}2.6 \%(3.6 \% \text { vs } 6.2 \% \\
\quad P=.008)\end{array}$ & $\begin{array}{l}0.5 \% \text { vs } 1.1 \%{ }^{\mathrm{d}} ; \\
\quad P=.21\end{array}$ \\
\hline CABG-CLARITY ${ }^{\mathrm{E} 33}$ & $\begin{array}{l}136 \text { STEMI patients } \\
\text { who underwent } \\
\text { CABG }\end{array}$ & $\begin{array}{l}\text { CLO (300 mg LD, } \\
75 \mathrm{mg} / \mathrm{d} \mathrm{MD}) \mathrm{vs} \\
\text { placebo for } 30 \mathrm{~d}\end{array}$ & $\begin{array}{l}\mathrm{CV} \text { death, } \mathrm{MI} \text {, or } \\
\text { recurrent ischemia } \\
\text { requiring urgent } \\
\text { revascularization }\end{array}$ & $\begin{array}{l}6.2 \%(15.2 \% \text { vs } 21.4 \% \\
\quad P=.37)\end{array}$ & $\begin{array}{l}7.6 \% \text { vs } 7.1 \%{ }^{\mathrm{d}} \\
\quad P=1.00\end{array}$ \\
\hline
\end{tabular}

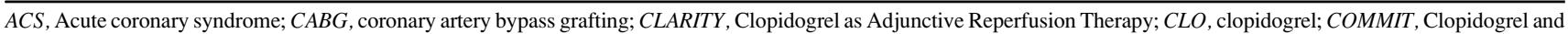
Metoprolol in Myocardial Infarction Trial; $C R E D O$, Clopidogrel for the Reduction of Events During Observation; CURE, Clopidogrel in Unstable Angina to Prevent Recurrent Events; $C V$, cardiovascular; $d$, day(s); $L D$, loading dose; $M D$, maintenance dose; $M I$, myocardial infarction; mo, month(s); PCI, percutaneous coronary intervention; STEMI, ST-elevation myocardial infarction; TIMI, Thromboylisis In Myocardial Infarction; wk, week(s). ${ }^{a}$ All patients received aspirin in addition to either clopidogrel, prasugrel, or placebo; ${ }^{b}$ CURE criteria for major bleeding (substantially disabling bleeding, intraocular bleeding leading to vision loss, bleeding necessitating transfusion of $\geq 2$ units of blood); ${ }^{\mathrm{c}} P$ value not provided; $95 \%$ confidence interval $(0.71-1.11) ;{ }^{\mathrm{d}}$ TIMI criteria for major bleeding (intracranial bleeding or bleeding associated with a hemoglobin drop $\geq 5 \mathrm{~g} / \mathrm{dL}$ ); ${ }^{\mathrm{e}}$ COMMIT criteria for major bleeding (fatal bleeding, cerebral bleeding, any bleeding that required transfusion). 


\section{E-References}

E1. Goldman S, Copeland J, Moritz T, Henderson W, Zadina K, Ovitt T, et al. Saphenous vein graft patency 1 year after coronary artery bypass surgery and effects of antiplatelet therapy. Results of a Veterans Administration Cooperative Study. Circulation. 1989;80:1190-7.

E2. Dacey LJ, Munoz JJ, Johnson ER, Leavitt BJ, Maloney CT, Morton JR, et al. Effect of preoperative aspirin use on mortality in coronary artery bypass grafting patients. Ann Thorac Surg. 2000;70:1986-90.

E3. Brown BG, Cukingnan RA, DeRouen T, Goede LV, Wong M, Fee HJ, et al. Improved graft patency in patients treated with platelet-inhibiting therapy after coronary bypass surgery. Circulation. 1985;72:138-46.

E4. Gavaghan TP, Gebski V, Baron DW. Immediate postoperative aspirin improves vein graft patency early and late after coronary artery bypass graft surgery. A placebo-controlled, randomized study. Circulation. 1991;83:1526-33.

E5. Mangano DT. Aspirin and mortality from coronary bypass surgery. $N$ Engl J Med. 2002;347:1309-17.

E6. Michelson EL, Morganroth J, Torosian M, MacVaugh H 3rd. Relation of preoperative use of aspirin to increased mediastinal blood loss after coronary artery bypass graft surgery. J Thorac Cardiovasc Surg. 1978;76:694-7.

E7. Ferraris VA, Ferraris SP, Lough FC, Berry WR. Preoperative aspirin ingestion increases operative blood loss after coronary artery bypass grafting. Ann Thorac Surg. 1988;45:71-4.

E8. Taggart DP, Siddiqui A, Wheatley DJ. Low-dose preoperative aspirin therapy, postoperative blood loss, and transfusion requirements. Ann Thorac Surg. 1990; 50:424-8.

E9. Goldman S, Copeland J, Moritz T, Henderson W, Zadina K, Ovitt T, et al. Improvement in early saphenous vein graft patency after coronary artery bypass surgery with antiplatelet therapy: results of a Veterans Administration Cooperative Study. Circulation. 1988;77:1324-32.

E10. Kallis P, Tooze JA, Talbot S, Cowans D, Bevan DH, Treasure T. Pre-operative aspirin decreases platelet aggregation and increases post-operative blood lossa prospective, randomised, placebo controlled, double-blind clinical trial in 100 patients with chronic stable angina. Eur J Cardiothorac Surg. 1994;8:404-9.

E11. Tuman KJ, McCarthy RJ, O'Connor CJ, McCarthy WE, Ivankovich AD. Aspirin does not increase allogeneic blood transfusion in reoperative coronary artery surgery. Anesth Analg. 1996;83:1178-84.

E12. Vuylsteke A, Oduro A, Cardan E, Latimer RD. Effect of aspirin in coronary artery bypass grafting. J Cardiothorac Vasc Anesth. 1997;11:831-4.

E13. Hongo RH, Ley J, Dick SE, Yee RR. The effect of clopidogrel in combination with aspirin when given before coronary artery bypass grafting. J Am Coll Cardiol. 2002;40:231-7.

E14. Englberger L, Faeh B, Berdat PA, Eberli F, Meier B, Carrel T. Impact of clopidogrel in coronary artery bypass grafting. Eur J Cardiothorac Surg. 2004;26: 96-101.

E15. Karabulut H, Toraman F, Evrenkaya S, Goksel O, Tarcan S, Alhan C. Clopidogrel does not increase bleeding and allogenic blood transfusion in coronary artery surgery. Eur J Cardiothorac Surg. 2004;25:419-23.

E16. Kapetanakis EI, Medlam DA, Boyce SW, Haile E, Hill PC, Dullum MK, et al. Clopidogrel administration prior to coronary artery bypass grafting surgery: the cardiologist's panacea or the surgeon's headache? Eur Heart J. 2005;26:576-83.

E17. Mehta RH, Roe MT, Mulgund J, Ohman EM, Cannon CP, Gibler WB, et al. Acute clopidogrel use and outcomes in patients with non-ST-segment elevation acute coronary syndromes undergoing coronary artery bypass surgery. J Am Coll Cardiol. 2006;48:281-6.
E18. Berger JS, Frye CB, Harshaw Q, Edwards FH, Steinhubl SR, Becker RC. Impact of clopidogrel in patients with acute coronary syndromes requiring coronary artery bypass surgery: a multicenter analysis. J Am Coll Cardiol. 2008;52:1693-701.

E19. Kim JH, Newby LK, Clare RM, Shaw LK, Lodge AJ, Smith PK, et al. Clopidogrel use and bleeding after coronary artery bypass graft surgery. Am Heart $\mathrm{J}$. 2008;156:886-92.

E20. Yende S, Wunderink RG. Effect of clopidogrel on bleeding after coronary artery bypass surgery. Crit Care Med. 2001;29:2271-5.

E21. Ray JG, Deniz S, Olivieri A, Pollex E, Vermeulen MJ, Alexander KS, et al. In creased blood product use among coronary artery bypass patients prescribed preoperative aspirin and clopidogrel. BMC Cardiovasc Disord. 2003;3:3.

E22. Leong JY, Baker RA, Shah PJ, Cherian VK, Knight JL. Clopidogrel and bleeding after coronary artery bypass graft surgery. Ann Thorac Surg. 2005;80:928-33.

E23. Nurozler F, Kutlu T, Kucuk G, Okten C. Impact of clopidogrel on postoperative blood loss after non-elective coronary bypass surgery. Interact Cardiovasc Thorac Surg. 2005;4:546-9.

E24. Hekmat K, Menzel C, Kroener A, Schwinger RH, Kampe S, Fischer UM, et al The effect of preoperative antiplatelet therapy in coronary artery surgery: blood transfusion requirements for patients on cardiopulmonary bypass. Curr Med Res Opin. 2004;20:1429-35.

E25. Shim JK, Choi YS, Oh YJ, Bang SO, Yoo KJ, Kwak YL. Effects of preoperative aspirin and clopidogrel therapy on perioperative blood loss and blood transfusion requirements in patients undergoing off-pump coronary artery bypass graft surgery. J Thorac Cardiovasc Surg. 2007;134:59-64.

E26. Yusuf S, Zhao F, Mehta SR, Chrolavicius S, Tognoni G, Fox KK. Effects of clopidogrel in addition to aspirin in patients with acute coronary syndromes without ST-segment elevation. N Engl J Med. 2001;345:494-502.

E27. Mehta SR, Yusuf S, Peters RJ, Bertrand ME, Lewis BS, Natarajan MK, et al Effects of pretreatment with clopidogrel and aspirin followed by long-term therapy in patients undergoing percutaneous coronary intervention: the PCI-CURE study. Lancet. 2001;358:527-33.

E28. Fox KA, Mehta SR, Peters R, Zhao F, Lakkis N, Gersh BJ, et al. Benefits and risks of the combination of clopidogrel and aspirin in patients undergoing surgical revascularization for non-ST-elevation acute coronary syndrome: the Clopidogrel in Unstable angina to prevent Recurrent ischemic Events (CURE) Trial. Circulation. 2004;110:1202-8.

E29. Steinhubl SR, Berger PB, Mann JT 3rd, Fry ET, DeLago A, Wilmer C, et al Early and sustained dual oral antiplatelet therapy following percutaneous coronary intervention: a randomized controlled trial. JAMA. 2002;288:2411-20.

E30. Chen ZM, Jiang LX, Chen YP, Xie JX, Pan HC, Peto R, et al. Addition of clopidogrel to aspirin in 45,852 patients with acute myocardial infarction: randomised placebo-controlled trial. Lancet. 2005;366:1607-21.

E31. Sabatine MS, Cannon CP, Gibson CM, Lopez-Sendon JL, Montalescot G, Theroux $\mathrm{P}$, et al. Addition of clopidogrel to aspirin and fibrinolytic therapy for myocardial infarction with ST-segment elevation. $N$ Engl J Med. 2005; 352:1179-89.

E32. Sabatine MS, Cannon CP, Gibson CM, Lopez-Sendon JL, Montalescot G, Theroux P, et al. Effect of clopidogrel pretreatment before percutaneous coronary intervention in patients with ST-elevation myocardial infarction treated with fibrinolytics: the PCI-CLARITY study. JAMA. 2005;294:1224-32.

E33. McLean DS, Sabatine MS, Guo W, McCabe CH, Cannon CP. Benefits and risks of clopidogrel pretreatment before coronary artery bypass grafting in patient with ST-elevation myocardial infarction treated with fibrinolytics in CLARITY-TIMI 28. J Thromb Thrombol. 2007;24:85-91. 